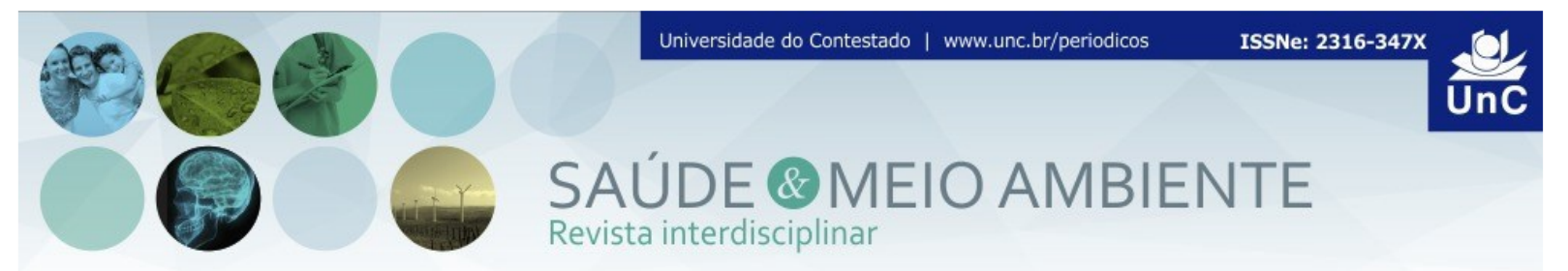

\title{
AVALIAÇÃO DA PERCEPÇÃO VISUAL COM O TEST OF VISUAL PERCEPTUAL SKILLS (TVPS-3) EM CRIANÇAS DE 6 A 14 ANOS COM TRANSTORNOS DE APRENDIZAGEM
}

\author{
Suellen Cristine Haensch ${ }^{1}$ \\ Angelita Fatima Beloto Dutra de Lima ${ }^{2}$
}

\begin{abstract}
RESUMO
INTRODUÇÃO: As habilidades perceptuais visuais fornecem a capacidade de organizar e interpretar estímulos visuais, dando sentido ao que é visto. Estes são atributos importantes para todas as situações de aprendizagem e contribuem com o desempenho acadêmico. OBJETIVOS: Avaliar através do teste TVPS-3 a percepção visual de alunos com idades entre 6 e 14 anos com transtornos de aprendizagem, tendo como objetivos específicos identificar quais habilidades perceptuais visuais se mostrarão alteradas, além de classificar através dos sinais e sintomas o transtorno específico e correlacionar as alterações das habilidades perceptuais visuais com o transtorno de aprendizagem. MATERIAIS E MÉTODOS: Estudo transversal, descritivo em 46 crianças com transtornos de aprendizagem. Os alunos foram avaliados com o teste TVPS-3, e os resultados foram apresentados em discriminação visual, memória visual, relação espacial, constância da forma, memória sequencial, figura-fundo, fechamento visual e pontuação global. As variáveis foram comparadas com os valores normais descritos na literatura. Foi realizado o teste de significância estatística utilizando a versão T-Student, bicaudal. A probabilidade de erro estatístico considerado foi igual a 5\%. RESULTADOS: A pontuação global em percentil apresentou valores abaixo ou muito abaixo da média em $98 \%$ da amostra. Os resultados dos testes de significância encontrados para discriminação visual foi de 0,925 , memória visual 0,959 , relação espacial 0,880 , constância da forma 0,917, memória sequencial 0,698, figura-fundo 0,834 e fechamento visual 0,888 . CONCLUSÕES: Os resultados estatísticos indicam uma forte relação entre os valores muito baixos encontrados no desempenho de cada habilidade de percepção visual e o transtorno de aprendizagem.
\end{abstract}

Palavras-Chave: Percepção Visual. Transtorno de Aprendizagem. Optometria

\footnotetext{
${ }^{1}$ Bacharel em Optometria, Pós Graduada em Alta Optometria, Especialista em Docência no Ensino Superior, Professora e Coordenadora do Curso de Optometria. Universidade do Contestado. Canoinhas. Santa Catarina. Brasil. E-mail: suellen@unc.br

${ }^{2}$ Bacharel em Optometria. Universidade do Contestado. Santa Catarina. Brasil. E-mail: angelitafraiburgo@hotmail.com
} 


\title{
EVALUATION OF VISUAL PERCEPTION WITH THE SKILLS VISUAL PERCEPTUAL TEST (TVPS-3) IN CHILDREN AGED 6 TO 14 YEARS WITH LEARNING DISORDERS
}

\begin{abstract}
INTRODUCTION: The visual perceptual skills provide the ability to organize, structure and interpret visual stimuli, giving meaning to what is seen. These are important attributes for all learning situations and contribute to academic performance. OBJECTIVES: To evaluate through TVPs-3 test visual perception of students aged 6 to 14 with learning disorders, with the specific objective to identify which visual perceptual skills will prove altered, and to classify the specific disorders through the signs and symptoms specific disorder and correlate changes in visual perceptual skills with the learning disorder. MATERIALS AND METHODS: Crosssectional, descriptive study in 46 children with learning disorders. Students were evaluated with TVPs-3 test, and the results were presented in visual discrimination, visual memory, spatial relationship, constancy of form, sequential memory, figureground, visual closure and overall score. The variables were compared with the standard values described in the literature. It conducted the statistical significance test using the T-Student version, two-tailed. The probability of statistical error considered was equal to $5 \%$. RESULTS: The overall score percentile values presented below, or well below the average in $98 \%$ of the sample. The results of significance tests found for visual discrimination was $0.925,0.959$ visual memory, spatial relationship 0.880 , constancy of shape $0.917,0.698$ sequential memory, figure-ground 0.834 and visual closure 0.888. CONCLUSIONS: The statistical results indicate a strong relationship between the very low values found in the performance of each skill of visual perception and learning disorder.
\end{abstract}

Keywords: Visual perception. Visual Perceptual Skills. Learning Disorder. Optometry.

\section{INTRODUÇÃO}

A percepção visual é a capacidade de processar estímulos visuais. É composta por um conjunto de habilidades perceptuais visuais essenciais para a leitura e o aprendizado, as quais se encarregam de analisar e processar a informação visual, obtendo assim uma compreensão do que se vê.

Atualmente, a importância dada aos problemas relacionados com a aprendizagem vem aumentando significativamente. As escolas apresentam um número considerável de alunos com transtornos de aprendizagem, e conforme descrito no Manual de Optometria Americana (2000), um grande percentual das crianças com transtornos de aprendizagem tem problemas de percepção visual, embora sua acuidade visual não esteja prejudicada em todos. 
Segundo Merchán (2008), dentro da pratica clinica da optometria pediátrica tem-se dado uma enorme importância em diagnosticar ametropias significativas, alterações na motilidade ocular ou alterações do tipo sensorial que podem levar à ambliopia ou que impeçam o desenvolvimento adequado de uma visão binocular, porém pouco está sendo levado em conta outros aspectos do sistema visual, como as habilidades de percepção visual. A avaliação optométrica pode ser útil no diagnóstico correto de pacientes com transtornos de aprendizagem, visando a atuação multidisciplinar.

Diante de um problema de transtornos na aprendizagem, os pais e profissionais relacionados com a educação e saúde, devem questionar-se quando os transtornos de aprendizagem são ocasionados por ineficiência das habilidades perceptuais visuais. A partir daí surge a seguinte pergunta: crianças com transtornos de aprendizagem apresentam as habilidades perceptuais visuais abaixo do padrão ou normais segundo o teste TVPS-3?

Um transtorno de aprendizagem, segundo Ohlweiler (2006) caracteriza uma inabilidade específica, como de leitura, escrita ou matemática, em indivíduos que apresentam resultados significativamente abaixo do esperado para seu nível de desenvolvimento, escolaridade e capacidade intelectual e que, de acordo com a Classificação Internacional de Doenças CID-10 (1992) e Clutten (2009) não apresentam problemas auditivos, visuais, sensoriais ou psicológicos importantes que possam explicar tais transtornos. Paín (1983), afirma que um transtorno de aprendizagem pode afetar a habilidade do indivíduo para ler, escrever, soletrar, raciocinar, recordar, organizar a informação ou aprender matemática, e que tais transtornos não devem ser confundidos com outras deficiências como o atraso mental, o autismo, a surdez, a cegueira ou os transtornos de comportamento. Nenhuma dessas deficiências constitui um transtorno de aprendizagem, tampouco deve ser confundido com a falta de oportunidades educativas.

Segundo a Organização Mundial de Saúde (OMS), a descrição dos transtornos de aprendizagem pode ser encontrada em Manuais Internacionais de Diagnósticos de Doenças CID-10 (1992) onde os transtornos são classificados em: F81.0- transtorno específico da leitura; F81.1- transtorno específico da ortografia; F81.2- transtorno específico do cálculo; F81.3- transtorno misto do desenvolvimento da aprendizagem escolar; F81.8- outros transtornos do desenvolvimento da aprendizagem escolar; F81.9- transtorno do desenvolvimento da aprendizagem escolar sem especificação.

Ler é uma tarefa que envolve os sistemas; sensorial, motor e cognitivo (Sousa, 2005). Rief e Heimburge's (2006) descrevem que, para um aluno obter sucesso na leitura, aspectos básicos de percepção visual são fundamentais. Habilidades acadêmicas básicas exigem tarefas de percepção visual, tais como a discriminação, o padrão e a relação de formas e objetos (LANDSBERG; KRUGER; NEL, 2005). Aprender a ler exige que o cérebro processe a informação em sete níveis diferentes (SOUSA, 2005), onde o aluno é obrigado a dominar habilidades específicas fundamentais (DEINER, 2005; FREDERICKSON; CLINE, 2006; GUNNING, 2006; SATTLER; WEYANDT, 2002). 
Conforme descrito pela Associação de Optometria Americana (2000), avaliado por Heath et al. (2006) e revisado por Garzia et al. (2008), nos problemas de visão relacionados com aprendizagem podem ocorrer déficits na eficiência visual e/ou no processamento da informação visual. A eficiência visual inclui erros refrativos não corrigidos, alterações vergenciais, acomodativas e na motilidade ocular. Os problemas no processamento da informação visual incluem deficiências nas habilidades de percepção visual, envolvendo funções superiores do cérebro, inclui aspectos de percepção visual e cognição, não motores, mas sim uma integração com o sistema visuomotor (BORSTING, 1996). Muitas vezes os problemas de visão relacionados com aprendizagem são interpretados como uma baixa da acuidade visual. Embora esta seja relevante para tarefas como copiar do quadro, outros aspectos da visão envolvendo a eficiência e o processamento da informação visual, são fundamentais para determinadas atividades como leitura, escrita e matemática (LERNER; KLINE, 2005).

Estudos realizados por Tallís e Soprano (2003) afirmam que as agnosias visuais englobam transtornos das funções perceptivas visuais e, segundo Dias e Chaves (2000) tais alterações na percepção visual podem explicar determinados transtornos que são observados nos processos de aprendizagem. Dednam (2005) descreve que quando um aluno tem déficit na percepção visual, tanto sua leitura como caligrafia e conceitos de matemática torna-se uma tarefa difícil, estes alunos realizam grande esforço resultando em fadiga, tarefas incompletas e déficit de concentração.

A percepção é um processo ativo de localização e extração da informação obtida do meio externo que se organiza em sistemas perceptuais, os quais realizam o processo de busca e obtenção da informação (BORSTING, 2006; MERCHÁN; CALDERÓN, 2011). A percepção visual é a capacidade do cérebro para organizar e interpretar o que é visto (DEINER, 2005; ERHARDT; DUCKMAN, 2005) e segundo Dednam (2005) e Giménez (2011) aproximadamente $80 \%$ do que se aprende é através da via visual, sendo assim a visão, o principal sistema perceptual de obtenção da informação.

Clutten (2009), Barry e Sargent (2006) argumentam que, para atender as demandas acadêmicas são necessárias habilidades de percepção visual, as quais são funções que facilitam a análise e o processamento da informação visual e são fundamentais para que um aluno reconheça primeiramente partes de formas, letras e números para finalmente reconhecer palavras, figuras e conceitos de matemática (SCHEIMAN; GALLAWAY, 2006). As habilidades perceptuais visuais estão subdivididas em discriminação visual: capacidade de discriminar características dominantes dos objetos, diferenciar posição, forma e cor, e permite ver a diferença entre objetos similares (MERCHÁN; CALDERÓN, 2011); memória visual: capacidade de reconhecer um estímulo depois de um breve intervalo de tempo (VASQUEZ; CUERVO, 2009); relação espacial: capacidade de orientar-se no espaço e perceber a posição dos objetos em relação a si mesmo e em relação com a posição relativa dos objetos (FROSTIG, 1993); constância da forma: capacidade para reconhecer um mesmo símbolo independentemente da orientação, forma ou tamanho (VASQUEZ; CUERVO, 2009); memória sequencial: capacidade para observar uma figura durante cinco segundos sendo capaz de reconhecê-la 
(VASQUEZ; CUERVO, 2009); figura-fundo: capacidade de identificar um objeto em um fundo complexo ou os objetos que se encontram ao redor (MERCHÁN, 2008); fechamento visual: capacidade de identificar uma figura em um conjunto, quando apenas partes das figuras são apresentadas (VASQUEZ; CUERVO, 2009).

Martin (2006) afirma que percepção e cognição caminham lado a lado e envolvem combinações de habilidades perceptuais visuais. Warren (1993) descreve que o processo de percepção visual começa com o estimulo visual e subsequentemente prossegue através de processos cognitivos superiores. Segundo Schneck (2005) e Carter (2012), o processo de construção da imagem começa quando a informação proveniente dos olhos, o material bruto, chega até o córtex visual primário na parte posterior do cérebro. Depois é enviada por duas vias principais, através das células parvo para a via ventral e células magno para a via dorsal, passando por áreas corticais e subcorticais. Cada uma responde criando uma atividade neural que gera aspectos da visão, como cor, forma, localização e movimento. Ao longo do trajeto percorrido pelas células magno, a atividade neuronal codifica a posição e o movimento do objeto. A via dorsal termina nas áreas parietais, que elaboram planos de ação relacionados ao objeto observado. Esse processo é inconsciente (CARTER, 2012), portanto, essa via está relacionada com a localização dos objetos, determina onde um objeto se encontra e conduz à ação (WURTZ; KANDEL, 2003). A via ventral é responsável por identificar o que é visto e dar um significado. Essa via segue uma rota que leva primeiro a uma série de áreas de processamento visual, cada uma acrescentando um aspecto específico da percepção, como forma, cor, contornos e detalhes finos. Portanto, a via ventral está relacionada com a identificação do que é visto, dando significado e conduzindo à percepção (WURTZ; KANDEL, 2003). A via ventral e a via dorsal têm funções diferentes, a informação é transmitida através de vias paralelas, porém separadas, e são reunidas no final nos lobos frontais, resultando em percepção consciente, e não em ação (WURTZ; KANDEL, 2003).

Para avaliar as habilidades de percepção visual foi desenvolvido por Gardner (1988) o teste TVPS, o qual foi aperfeiçoado e já está em sua terceira edição TVPS3 que, segundo Martin (2006), avalia as habilidades perceptuais visuais sem a necessidade do envolvimento de habilidades motoras quando fizer uma resposta. As habilidades motoras e perceptivas visuais se desenvolvem em paralelo e estão intimamente relacionadas, porém os dois sistemas são de fato distintos (LEONARD; FOXCRAFT; KROUKAMP, 1988; PARUSH, et al., 1998). Newcomer e Hammill (1973), Schneck (2005) e Clutten (2009) descrevem que uma criança que tem um problema motor não tem necessariamente um problema de percepção visual. De acordo com estudos realizados por Vasquez e Cuervo (2009), o TVPS-3 é mais completo quanto à estrutura, organização e resultados comparando com outros testes similares, como o MVPT, Beery-VMI, DTVP, contendo estes, componente visuomotor quando se realiza uma resposta e por avaliarem as habilidades de percepção visual de uma maneira global. Clinicamente, para a avaliação da percepção visual, os autores sugerem utilizar o teste TVPS-3 por ser mais específico, pois avalia individualmente as habilidades perceptuais visuais, dando uma informação mais confiável sobre o sistema visuoperceptual da criança. 
Zhong-Lin et al. (2010) definem a aprendizagem visuoperceptual como um fenômeno geral relacionado com processos visuais melhorados como resultado de treinamento ou da prática de tarefas perceptuais visuais, e é considerado como uma forma de plasticidade neuronal que tem consequências no córtex visual (BUONOMANO; MERZENICH, 1998). Vários estudos, realizados por Gilbert, Sigman e Crist (2001); Adini et al. (2004); Fahle (2005); Xiao et al. (2008) Levi e Li (2009); Polat, Ma-Naim e Spierer (2009); tem demonstrado a relação que existe entre o treinamento das habilidades perceptuais visuais e a melhoria da aprendizagem.

Este estudo objetiva avaliar, através do teste TVPS-3, a percepção visual de alunos com transtornos de aprendizagem, tendo como objetivos específicos, identificar quais habilidades de percepção visual se mostrarão alteradas, além de classificar através dos sinais e sintomas o transtorno específico, correlacionando assim, as alterações das habilidades perceptuais visuais com o transtorno de aprendizagem.

\section{PROCEDIMENTOS METODOLÓGICOS}

Participaram deste estudo transversal, observacional descritivo 78 crianças com transtornos de aprendizagem, com idades entre 6 e 14 anos, que não apresentavam deficiência física, mental, auditiva e/ou visual causada por patologias, entre outras deficiências associadas ao transtorno de aprendizagem e, também, as que não faziam uso de medicamentos que alteram o sistema nervoso central. O responsável legal pelo aluno assinou um termo de assentimento livre e esclarecido para menores. Os alunos foram avaliados com os seguintes testes: acuidade visual, oftalmoscopia, retinoscopia estática e dinâmica, subjetivo, kappa, hirschberg, ppc, cover test, aca, mem, estereopsia e movimentos oculomotores. Foram excluídos da amostra, alunos que apresentaram ambliopias, estrabismos, altas ametropias, problemas acomodativos, motores e/ou vergenciais. Após a seleção da amostra, de acordo com os critérios de exclusão, 46 alunos foram avaliados com o teste TVPS-3. A classificação dos sinais e sintomas foi realizado pela psicóloga da escola. A pesquisa teve aprovação do Comitê de Ética em Pesquisa (CEP) da Universidade do Contestado, conforme número do parecer 1.019 .173 de 07/05/2015. Os resultados foram descritos de acordo com as seguintes habilidades perceptuais visuais: discriminação visual, memória visual, relação espacial, constância da forma, memória sequencial, figura-fundo, fechamento visual e pontuação global. As variáveis foram comparadas com os valores normais descritos na literatura e foi realizado o teste de significância estatística utilizando a versão T-Student. 


\section{RESULTADOS E DISCUSSÕES}

Este capítulo apresenta os resultados da pesquisa, mostrando inicialmente a classificação do transtorno específico de aprendizagem, no qual cada aluno se encontra, assim como o desempenho da amostra em cada habilidade de percepção visual, e também os valores globais do desempenho dos alunos. Por fim, para cumprir os objetivos da pesquisa, apresenta-se o desempenho dos alunos classificados no transtorno misto do desenvolvimento da aprendizagem escolar em cada habilidade de percepção visual, correlacionando as alterações das habilidades perceptuais visuais com o transtorno específico de aprendizagem apresentado.

Tabela 1 - Classificação dos transtornos de aprendizagem segundo a Organização Mundial de Saúde (OMS) e a Classificação Internacional de Doenças (CID-10)

\begin{tabular}{|c|c|c|}
\hline Transtorno apresentado & $\mathrm{n}^{\circ}$ alunos & Percenti \\
\hline Transtorno misto do desenvolvimento da aprendizagem escolar & 45 & $97,83 \%$ \\
\hline Transtorno específico da leitura & 01 & $02,17 \%$ \\
\hline Total de alunos & 46 & $100,00 \%$ \\
\hline
\end{tabular}

Fonte: Dados da pesquisa (2015)

Referente à Classificação dos Transtornos de Aprendizagem da amostra, na tabela 1, dos 46 alunos avaliados pelo TVPS-3, 98\% encontram-se dentro da Classificação do Transtorno Misto do Desenvolvimento da Aprendizagem Escolar, no qual, segundo a Classificação Internacional de Doenças CID-10 (1992) estão alterados tanto o rendimento matemático como o da leitura ou ortografia e no qual a inteligência geral está dentro da classe normal e não está presente um mau ensino escolar, e $02 \%$ encontram-se dentro do Transtorno Específico da Leitura, onde existe um déficit específico do desenvolvimento da capacidade de ler que não é explicado pelo nível intelectual, por problemas de acuidade visual ou por uma escolarização inadequada. Podem ser afetadas a capacidade de compreensão da leitura, o reconhecimento de palavras lidas, a capacidade de ler em voz alta e o rendimento em atividades que exigem a leitura (OMS-CID-10, 1992).

Tabela 2 - Desempenho em cada habilidade perceptual visual dos 45 alunos classificados no transtorno misto do desenvolvimento da aprendizagem escolar

\begin{tabular}{lrrrrrrr}
\hline Desempenho & \multicolumn{1}{c}{ DIS } & MEM & \multicolumn{1}{c}{ SPA } & \multicolumn{1}{c}{ CON } & \multicolumn{1}{c}{ SEQ } & \multicolumn{1}{l}{ FGR } & \multicolumn{1}{c}{ CLO } \\
\hline Muito baixo & $55,57 \%$ & $42,23 \%$ & $48,91 \%$ & $68,90 \%$ & $28,91 \%$ & $55,58 \%$ & $53,35 \%$ \\
Baixo & $35,55 \%$ & $51,11 \%$ & $35,55 \%$ & $24,44 \%$ & $48,88 \%$ & $26,66 \%$ & $33,33 \%$ \\
Médio/normal & $2,22 \%$ & $0,00 \%$ & $6,66 \%$ & $6,66 \%$ & $15,55 \%$ & $6,66 \%$ & $6,66 \%$ \\
Alto & $4,44 \%$ & $6,66 \%$ & $8,88 \%$ & $0,00 \%$ & $6,66 \%$ & $8,88 \%$ & $0,00 \%$ \\
Superior & $2,22 \%$ & $0,00 \%$ & $0,00 \%$ & $0,00 \%$ & $0,00 \%$ & $2,22 \%$ & $6,66 \%$ \\
\hline Total & $\mathbf{1 0 0} \%$ & $\mathbf{1 0 0} \%$ & $\mathbf{1 0 0} \%$ & $\mathbf{1 0 0} \%$ & $\mathbf{1 0 0} \%$ & $\mathbf{1 0 0} \%$ & $\mathbf{1 0 0} \%$ \\
\hline
\end{tabular}

Legenda: DIS - discriminação visual, MEM - memória visual, SPA - relação espacial, CON constância da forma, SEQ - memória sequencial, FGR - figura-fundo, CLO - fechamento visual Fonte: dados da pesquisa (2015) 
Gráfico 1 - Desempenho em cada habilidade perceptual visual dos 45 alunos classificados no transtorno misto do desenvolvimento da aprendizagem escolar

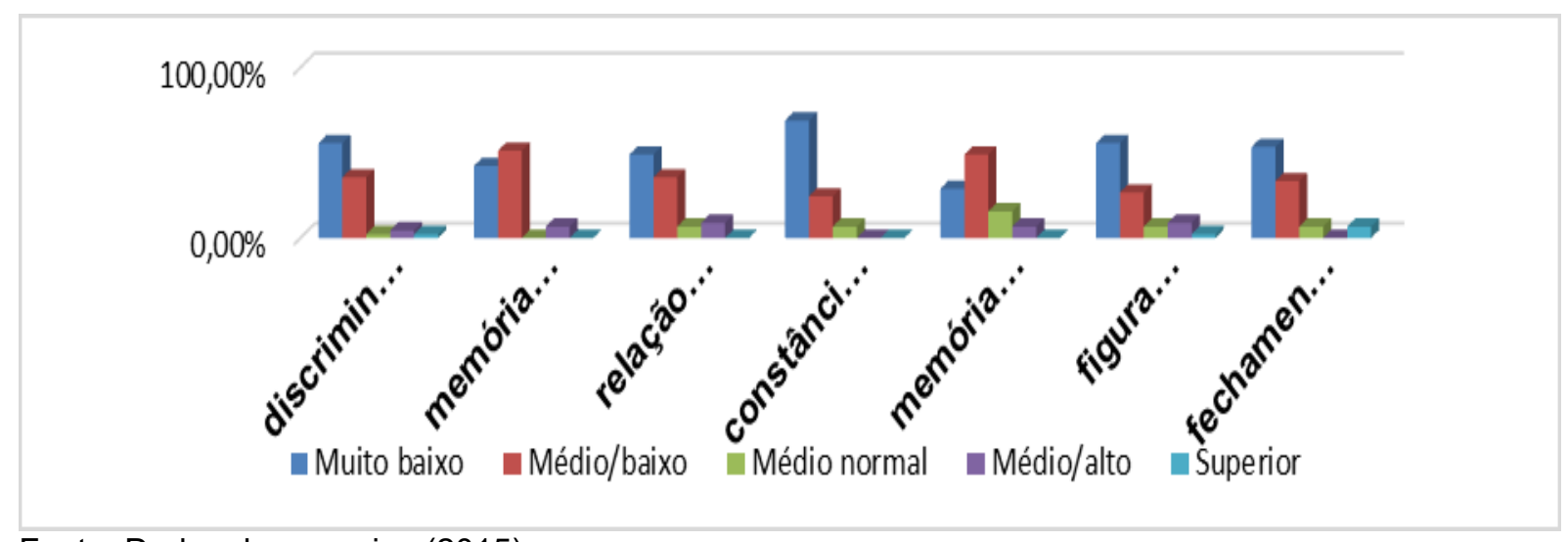

Fonte: Dados da pesquisa (2015)

Analisando na tabela 2 e gráfico 1, o desempenho dos 45 alunos classificados no transtorno misto do desenvolvimento da aprendizagem escolar, $91 \%$ apresentaram a habilidade discriminação visual abaixo ou muito abaixo da média, $93 \%$ apresentaram a habilidade memória visual abaixo ou muito abaixo da média, $84 \%$ apresentaram a habilidade relação espacial abaixo ou muito abaixo da média, 93\% apresentaram a habilidade constância da forma abaixo ou muito abaixo da média, $78 \%$ apresentaram a habilidade memória sequencial abaixo ou muito abaixo da média, $82 \%$ apresentaram a habilidade figura-fundo abaixo ou muito abaixo da média e $87 \%$ apresentaram a habilidade fechamento visual abaixo ou muito abaixo da média. Observa-se que $100 \%$ dos alunos obtiveram valores abaixo de $50 \%$ em todas as habilidades perceptuais visuais, considerando que, para Martin (2006), para que um aluno esteja dentro da média deverá ter uma pontuação em percentil de $50 \%$ ou mais.

Tabela 3 - Desempenho dos 46 alunos na habilidade perceptual visual sobre Discriminação Visual

\begin{tabular}{clcccc}
\hline \% de Rank & Classificação & $\mathbf{n}^{\circ}$ alunos & Fia & Fr & Fra \\
\hline $1 \%<15 \%$ & Muito baixo & 26 & 26 & $56,53 \%$ & $56,53 \%$ \\
$16 \%>49 \%$ & Baixo/médio & 16 & 42 & $34,78 \%$ & $91,30 \%$ \\
$50 \%$ & Médio/normal & 01 & 43 & $02,17 \%$ & $93,48 \%$ \\
$51 \%>84 \%$ & Médio/alto & 02 & 45 & $04,35 \%$ & $97,83 \%$ \\
$85 \%>99 \%$ & Superior & 01 & 46 & $02,17 \%$ & $100,00 \%$ \\
\hline
\end{tabular}

Fonte: Dados da pesquisa (2015)

A habilidade perceptual visual sobre Discriminação Visual dos alunos avaliados com o TVPS-3 mostrou-se abaixo ou muito abaixo da média em $91 \%$ da amostra. De acordo com Kurtz (2003) e Dednam (2005), sendo a discriminação visual uma habilidade a qual permite que a criança diferencie figuras simples como $6 / 9,7 / 1,12 / 21, \mathrm{f} / \mathrm{p}, \mathrm{n} / \mathrm{m}$, b/d, dificuldades nessa habilidade podem causar confusão de palavras similares, dificuldades em perceber semelhanças, diferenças e erros ao ler palavras com inícios e finais parecidos. 
Tabela 4 - Desempenho dos 46 alunos na habilidade perceptual visual sobre Memória Visual

\begin{tabular}{clcccc}
\hline$\%$ de Rank & Classificação & $\mathbf{n}^{\circ}$ alunos & Fia & Fr & Fra \\
\hline $1 \%<15 \%$ & Muito baixo & 19 & 19 & $41,31 \%$ & $41,31 \%$ \\
$16 \%>49 \%$ & Baixo/médio & 24 & 43 & $52,17 \%$ & $93,48 \%$ \\
$50 \%$ & Médio/normal & 0 & 43 & $0,00 \%$ & $93,48 \%$ \\
$51 \%>84 \%$ & Médio/alto & 3 & 46 & $6,52 \%$ & $100,00 \%$ \\
$85 \%>99 \%$ & Superior & 0 & 46 & $0,00 \%$ & $100,00 \%$ \\
\hline
\end{tabular}

Fonte: Dados da pesquisa (2015)

Conforme sumariado na tabela, $93 \%$ dos alunos apresentaram valores abaixo ou muito abaixo da média para a habilidade sobre Memória Visual. Sattler (2002) e Borsting (2006) descrevem que o desenvolvimento de uma boa memória visual é um aspecto básico na aprendizagem, pois permite que um aluno retenha estímulos visuais observados, já que é uma habilidade para recordar as características completas de uma figura em um conjunto. Kavale (1982) ainda descreve que a memória visual é um aspecto importante para a leitura, ortografia e para resolver problemas matemáticos.

Tabela 5 - Desempenho dos 46 alunos na habilidade perceptual visual sobre Relação Espacial

\begin{tabular}{clcccc}
\hline \% de Rank & Classificação & n $^{\circ}$ alunos & Fia & Fr & Fra \\
\hline $1 \%<15 \%$ & Muito baixo & 22 & 22 & $47,83 \%$ & $47,83 \%$ \\
$16 \%>49 \%$ & Baixo/médio & 17 & 39 & $36,96 \%$ & $84,78 \%$ \\
$50 \%$ & Médio/normal & 3 & 42 & $6,52 \%$ & $91,30 \%$ \\
$51 \%>84 \%$ & Médio/alto & 4 & 46 & $8,70 \%$ & $100,00 \%$ \\
$85 \%>99 \%$ & Superior & 0 & 46 & $0,00 \%$ & $100,00 \%$ \\
\hline
\end{tabular}

Fonte: Dados da pesquisa (2015)

O resultado da avaliação para a habilidade perceptual visual sobre relação espacial apresentou-se abaixo ou muito abaixo da média em $85 \%$ dos alunos avaliados, essa habilidade segundo Hammill, Pearson e Voress (1993) e Schneck (2005), é a capacidade de avaliar com precisão a relação física entre o corpo e o meio ambiente ou a relação física entre dois objetos, incluindo a compreensão das mudanças dessas relações físicas com o movimento. Williams (1983), Cheatum e Hammond (2000), relatam que a orientação espacial tem um papel importante no desenvolvimento da leitura e da escrita, porque ambas as atividades estão imersas em uma direção muito específica, sendo da esquerda para a direita. Ao escrever, todas as letras seguem a mesma direção sendo fácil entrelaçá-las fazendo que sejamos mais ágeis escrevendo. Quando uma criança não tem certeza dessa direção aparecem as inversões na leitura ou as rotações de letras simétricas que podem provocar deficiência na lectoescritura que, em algumas ocasiões, podem se confundir com dislexia. A interpretação de mapas também pode ser difícil para pessoas com má orientação espacial. 
Avaliação da percepção visual com o Test Of Visual Perceptual SKILLS (TVPS-3) em crianças de 6 a 14 anos com transtornos de aprendizagem

Tabela 6 - Desempenho dos 46 alunos na habilidade perceptual visual sobre Constância da Forma

\begin{tabular}{clcccc}
\hline$\%$ de Rank & Classificação & $\mathbf{n}^{\circ}$ alunos & Fia & Fr & Fra \\
\hline $1 \%<15 \%$ & Muito baixo & 31 & 31 & $67,39 \%$ & $67,39 \%$ \\
$16 \%>49 \%$ & Baixo/médio & 13 & 44 & $28,26 \%$ & $95,65 \%$ \\
$50 \%$ & Médio/normal & 2 & 46 & $4,35 \%$ & $100,00 \%$ \\
$51 \%>84 \%$ & Médio/alto & 0 & 46 & $0,00 \%$ & $100,00 \%$ \\
$85 \%>99 \%$ & Superior & 0 & 46 & $0,00 \%$ & $100,00 \%$ \\
\hline
\end{tabular}

Fonte: Dados da pesquisa (2015)

Na tabela 6 observa-se o desempenho das crianças na habilidade perceptual visual sobre Constância da Forma, na qual 96\% dos alunos encontram-se abaixo ou muito abaixo da média para essa habilidade que, segundo Schneck (2005) e Dednam (2005), é a capacidade de reconhecer um mesmo símbolo independentemente da orientação, forma ou tamanho. Quando esta habilidade está afetada pode-se encontrar dificuldades para reconhecer o mesmo símbolo escrito de diferentes maneiras (CHEATUM; HAMMOND, 2000; DEDNAM, 2005).

Tabela 7 - Desempenho dos 46 alunos na habilidade perceptual visual sobre Memória Seqüencial

\begin{tabular}{clcccc}
\hline \% de Rank & Classificação & $\mathbf{n}^{\circ}$ alunos & Fia & Fr & Fra \\
\hline $1 \%<15 \%$ & Muito baixo & 13 & 13 & $28,26 \%$ & $28,26 \%$ \\
$16 \%>49 \%$ & Baixo/médio & 21 & 34 & $45,65 \%$ & $73,91 \%$ \\
$50 \%$ & Médio/normal & 7 & 41 & $15,22 \%$ & $89,13 \%$ \\
$51 \%>84 \%$ & Médio/alto & 5 & 46 & $10,87 \%$ & $100,00 \%$ \\
$85 \%>99 \%$ & Superior & 0 & 46 & $0,00 \%$ & $100,00 \%$ \\
\hline
\end{tabular}

Fonte: Dados da pesquisa (2015)

A tabela 7 apresentam o desempenho dos alunos na habilidade perceptual visual sobre Memória Sequencial, onde pode-se observar que $74 \%$ da amostra apresentou valores abaixo ou muito abaixo da média. Com essa habilidade avaliamos a capacidade para memorizar uma sequência, permitindo memorizar as letras que formam uma determinada palavra e arquivá-la para quando vejamos novamente possamos reconhecê-la, associá-la a um significado e expressá-la verbalmente (SCHEIMAN; ROUSE, 2006). Rief e Heimburge's (2006) descrevem que este aspecto perceptual visual é crucial para a leitura, escrita, ortografia e matemática, relatando ainda que para a leitura, ocorrem frequências de palavras que não são fonéticas, sendo estas aprendidas por reconhecimento visual, portanto, alunos com déficit na memória sequencial tendem a sussurrar enquanto escrevem e tarefas como a cópia de frases ou a aprendizagem de palavras não usadas habitualmente como os ossos do corpo humano ou países e capitais tornam-se muito difíceis.

Tabela 8 - Desempenho dos 46 alunos na habilidade perceptual visual sobre Figura-Fundo

\begin{tabular}{clcccc}
\hline \% de Rank & Classificação & $\mathbf{n}^{\circ}$ alunos & Fia & Fr & Fra \\
\hline $1 \%<15 \%$ & Muito baixo & 25 & 25 & $54,35 \%$ & $54,35 \%$ \\
$16 \%>49 \%$ & Baixo/médio & 13 & 38 & $28,26 \%$ & $82,61 \%$ \\
$50 \%$ & Médio/normal & 3 & 41 & $6,52 \%$ & $89,13 \%$ \\
$51 \%>84 \%$ & Médio/alto & 4 & 45 & $8,70 \%$ & $97,83 \%$ \\
$85 \%>99 \%$ & Superior & 1 & 46 & $2,17 \%$ & $100,00 \%$ \\
\hline
\end{tabular}

Fonte: Dados da pesquisa (2015) 
Considerando os resultados apresentados conforme tabela 8, nota-se um desempenho abaixo ou muito abaixo da média em $83 \%$ dos alunos na habilidade perceptual visual sobre figura-fundo. Esta habilidade permite detectar, diferenciar e selecionar estímulos visuais dentro de um determinado entorno. É o que permite diferenciar um objeto de atenção dos outros presentes no campo visual e também permite extrair de um contexto visual a informação importante, sendo o aluno capaz de perceber e localizar uma forma ou objeto dentro de um campo ocupado sem ser confundido pelo fundo ou imagens ao redor (LERNER, 2000). A criança com dificuldades nessa área perceptiva tem dificuldades na compreensão da informação, elas ficam perdidas com os detalhes de uma figura estando distraídas por estímulos irrelevantes e se confundem facilmente ao ler textos com muitas letras e tem dificuldades para localizar informações específicas dentro de um texto, afetando os níveis de concentração e atenção (DURÁN, 2009; DEDNAM, 2005).

Tabela 9 - Desempenho dos 46 alunos na habilidade perceptual visual sobre Fechamento Visual

\begin{tabular}{clcccc}
\hline$\%$ de Rank & Classificação & $\mathbf{n}^{\circ}$ alunos & Fia & Fr & Fra \\
\hline $1 \%<15 \%$ & Muito baixo & 25 & 25 & $54,35 \%$ & $54,35 \%$ \\
$16 \%>49 \%$ & Baixo/médio & 15 & 40 & $32,61 \%$ & $86,96 \%$ \\
$50 \%$ & Médio/normal & 3 & 43 & $6,52 \%$ & $93,48 \%$ \\
$51 \%>84 \%$ & Médio/alto & 0 & 43 & $0,00 \%$ & $93,48 \%$ \\
$85 \%>99 \%$ & Superior & 3 & 46 & $6,52 \%$ & $100,00 \%$ \\
\hline
\end{tabular}

Fonte: Dados da pesquisa (2015)

A análise do desempenho da habilidade sobre fechamento visual conforme tabela 9 mostra valores abaixo ou muito abaixo da média em $87 \%$ dos alunos. Considerando, segundo Kavale (1982) e Lerner (2000), ser essa habilidade a capacidade para identificar e reconhecer um objeto quando falta parte dele, o fechamento visual permite uma leitura rápida e de fácil compreensão. De acordo com Dednam (2005) o processamento visual não tem que processar cada letra em cada palavra para reconhecê-la, quando apenas uma parte é suficiente. Os alunos com deficit no fechamento visual enfrentam desafios na leitura global, escrita, ortografia e na compreensão, bem como complicações em completar um pensamento, segundo Dednam (2005).

Tabela 10 - Valores do desempenho global dos 46 alunos, envolvendo todas as habilidades perceptuais visuais avaliadas através do teste TVPS-3

\begin{tabular}{clccccc}
\hline \% de Rank & Classificação & Pontuação global & $\mathbf{n}^{\circ}$ alunos & Fia & Fr & Fra \\
\hline $1 \%<15 \%$ & Muito baixo & $0-84$ & 29 & 29 & $63,05 \%$ & $63,04 \%$ \\
$16 \%>49 \%$ & Baixo/médio & $85-99$ & 16 & 45 & $34,78 \%$ & $97,83 \%$ \\
$50 \%$ & Médio/normal & 100 & 0 & 45 & $0,00 \%$ & $97,83 \%$ \\
$51 \%>84 \%$ & Médio/alto & $101-115$ & 1 & 46 & $2,17 \%$ & $100,00 \%$ \\
$85 \%>99 \%$ & Superior & $116-145$ & 0 & 46 & $0,00 \%$ & $100,00 \%$ \\
\hline
\end{tabular}

Fonte: Dados da pesquisa (2015) 
Conforme demonstra a tabela 10, a pontuação global em percentil dos 46 alunos avaliados apresentou valores globais abaixo ou muito abaixo da média em $98 \%$ da amostra. Considerando estudos realizados por Scott (2003), Gordon (2004), Dednam (2005), Scheiman e Rouse (2006), Rief e Heimburge's (2006), Beery e Beery (2006), todos consideram que há uma significativa correlação entre déficits na percepção visual e a capacidade de um aluno para aprender, onde desafios de aprendizagem têm sido frequentemente relacionados à deficiências nos aspectos visuais que envolvem as habilidades de percepção visual. Os autores discutem questões relacionadas com a importância da percepção visual para a aprendizagem, considerando a percepção visual fundamental para garantir que um aluno realize de forma adequada várias atividades escolares.

Por fim, foi realizado o teste de significância estatística utilizando a versão TStudent, para comparar os valores obtidos entre o desempenho de cada habilidade perceptual visual com o transtorno específico de aprendizagem. Foi utilizado o teste bicaudal, considerando erro estatístico de $5 \%$.

Abaixo apresentam-se os resultados obtidos.

Tabela 11 - Resultados obtido através do teste de significância estatística T - Student

\begin{tabular}{ccccccc}
\hline DIS & MEM & SPA & COM & SEQ & FGR & CLO \\
0,925 & 0,959 & 0,880 & 0,917 & 0,698 & 0,834 & 0,888 \\
\hline
\end{tabular}

Fonte: Dados da pesquisa (2016)

Conforme os resultados do teste, demonstrados na tabela 11, temos todos os valores localizados entre $-1,679$ e $+1,679$. Esta condição indica que as médias estão próximas, e que, a hipótese nula não deve ser descartada, ou seja, nota-se uma grande semelhança entre os resultados, indicando uma forte relação entre os valores abaixo, e/ou muito abaixo da média encontrados no desempenho de cada habilidade de percepção visual e o transtorno misto do desenvolvimento da aprendizagem escolar.

\section{CONCLUSÃO}

De acordo com os resultados obtidos, foi possível identificar quais habilidades perceptuais visuais se encontram ineficientes, onde $91 \%$ dos alunos apresentaramse com valores abaixo da média na discriminação visual, 93\% apresentaram a habilidade memória visual abaixo da média, $85 \%$ apresentaram a habilidade relação espacial abaixo da média, $96 \%$ apresentaram a habilidade constância da forma abaixo da média, $74 \%$ apresentaram a habilidade memória sequencial abaixo da média, $83 \%$ apresentaram a habilidade figura-fundo abaixo da média e $87 \%$ apresentaram a habilidade fechamento visual abaixo da média, assim como o desempenho nos valores globais, no qual $98 \%$ dos alunos obtiveram pontuações abaixo da média. Observa-se que de $98 \%$ dos alunos classificados no transtorno misto do desenvolvimento da aprendizagem escolar, todos apresentaram valores 
abaixo da média para todas as habilidades de percepção visual. Correlacionando as alterações das habilidades perceptuais visuais com o transtorno misto do desenvolvimento da aprendizagem escolar, e conforme demonstrado no teste de significância estatística, a hipótese nula, ou seja, a relação existente entre os transtornos de aprendizagem com a ineficiência das habilidades de percepção visual não pode ser descartadas, indicando que, quando a percepção visual de um aluno é ineficiente, pode contribuir negativamente para o processo de aprendizagem.

Os resultados sugerem a importância de uma avaliação não somente da eficiência visual, mas também da capacidade das habilidades de percepção visual no processamento da informação visual de crianças com transtornos de aprendizagem. Portanto, a avaliação optométrica pode ser útil, dentro de uma equipe multidisciplinar de profissionais da saúde e educação, tanto no diagnóstico como na reabilitação das habilidades perceptuais visuais, com atividades que propiciem $o$ desenvolvimento das habilidades necessárias para um bom desenvolvimento da leitura, escrita e matemática. Sugere-se também, um estudo sobre a reabilitação das alterações nas habilidades perceptuais visuais.

\section{REFERÊNCIAS}

Adini Y, Wilkonsky A, Haspel R, Tsodyks S, Misha S, Dov S. Perceptual learning in contrast discrimination: The effect of contrast uncertainty. Journal of Vision; 2004; 4 (12):993-1005.

Optometric clinical practice guideline: Care of the patient with learning related vision problems [Internet]. American Optometric Association; 2000 [cited 15 March 2019]. Available from: https://www.aoa.org/documents/optometrists/CPG-20.pdf

Vision training [Internet]. Optometrists; 2006 [cited 21 November 2015]. Available from: http://www.barryandsargent.co.nz/children.html

Beery KE, Beery NA. The Beery-Buktenica developmental test of Visual Motor Integration (Beery VMI). Minneapolis: Pearson; 2006.

Borsting EJ. Visual Perception and Reading. In: G. R. Vision Reading. California: Mosby; 1996, 149-176.

Borsting EJ. Overview of vision efficiency and visual processing development. In: M. S, M. R, Optometric management of learning related vision problems. Boston: Mosby; 2006, 35-68.

Buonomano DV.; Merzenich MM. Cortical Plasticity: from synapses to maps. Annual Review of Neuroscience; 2019, 21: 149-186.

Carter R. O livro do cérebro. Rio de Janeiro: Agir; 2012. 
Cheatum BA, Hammond A. Physical activities for improving children's learning and behavior. Champaign: Human Kinetics; 2000.

Organização Mundial da Saúde (OMS). CID-10. Classificação Estatística internacional de Doenças e Problemas Relacionados à Saúde; 1992.

Clutten SC. The development of a visual perception test for learners in the foundation phase. University of South Africa; 2009.

Dednam A. Learning impairment. In LANDSBERG, Emmerentia; KRUGER, Deirdré; NEL, Norma. Addressing barriers to learning: a south African perspective. Van Schaik; 2005, 363-379.

Deiner PL. Resources for educating children with diverse abilities. New York: Thomson Delmar Learning; 2005, 4.

Dias MMCBS, Chaves JHSS. Percepção visual e dificuldades de aprendizagem: um estudo com alunos do $1^{\circ}$ ciclo do ensino básico. Revista Galego-Portuguesa de Psicologia e Educação. Portugal: Universidade da Coruña; 2000; 6:389-398.

Durán MM. Training activities for visual perceptual skills. Saera: Solutions for learning and research; 2009.

Erhardt RP, Duckman RH. Visual-perceptual-motor dysfunction: effects of eye-hand coordination and skills development. Bathesda: The American Occupational Therapy Associaton; 2005; 2.

Fahle M. Perceptual learning: specificity versus generalization. Current Opinion in Neurobiology. Elsevier Current Trends; 2005, 12 (2): 154-160.

Frederickson N, Cline T. Special educational needs, inclusion and diversity. Glasgow: Open University Press; 2006.

Frostig M. Discapacidades específicas de aprendizaje en niños. Buenos Aires: Medica Panamericana; 1993; 2.

Gardner MF. Test of visual perceptual skills (non-motor) TVPS manual. San Francisco: Health Publishing Company; 1988.

Garzia RP, Borsting EJ, Nicholson SB, Press LJ, Scheiman MM, Solan HA. Optometric clinical practice guideline care of the patient with learning related vision problems.St Louis: American Optometric Association; 2008.

Gilbert CD, Sigman M, Crist RE. The neural basis of perceptual learning. Neuron. Sitges: Spain; 2001, 31: 681-697

Giménez MPV. Tanta inteligência, tão pouco rendimento. São Paulo: Conceito; 2011.

Gordon IE. Theories of visual perception. New York: Psychology Press; 2004, 3. 
Gunning TG. Assessing and correcting reading and writing difficulties. Boston: Pearson Education Inc.; 2006, 3.

Hammill DD, Pearson NA, Voress JK. Developmental test of visual perception. Austin, Pro; 1993, 2.

Heath DA, Amos JF, Miller SC. Optometric clinical practice guideline care of the patient with learning related vision problems. St. Louis: American Optometric Association; 2006.

Kavale KA. Meta-Analysis of the relationship between visual perceptual skills and reading achievements. Journal of Learning Disabilities; 1982, 15: 42-51.

Kurtz LA. How to help a clumsy child: strategies for young children with developmental motor concerns. London: Jessica Kingsley Publishers; 2003.

Landsberg E, Kruger D, Nel N. Addressing barriers to learning: a south African perspective. Van Schaik Publishers; 2005.

Leonard P, Foxcroft C, Kroukamp T. Are visual perceptual and visual motor skills separate abilities? Perceptual and Motor skills. University of Port Elizabeth; 1988, 67: 423-426.

Lerner JW, Kline F. Learning disabilities and related disorders: characteristics and teaching strategies. Boston: Houghton Mifflin; 2005, 10.

Lerner JW. Learning disabilities: theories, diagnosis and teaching strategies. Boston: Houghton Mifflin; 2000, 8.

Levi DM, Li RW. Perceptual learning as a potential treatment for amblyopia: a mini review. Vision Research; 2009, 49: 2535-2549.

Martin NA. Test of visual perceptual skills. Novato: Academic Therapy Publications; 2006, 3.

Merchán MSP, Calderón JLH. Influencia de la percepción visual en el aprendizaje. Bogotá: Universidad de La Salle; 2011.

Merchán MSP. Relación causa-efecto entre ametropías altas y habilidades perceptuales visuales. Bogotá: Universidad de La Salle; 2008.

Newcomer P, Hammill DD. Visual perception of motor impaired children: implications for assessment. Exceptional Children: Academic Journal; 1973, 39: 335-337.

Ohlweiler L. Transtornos da aprendizagem: abordagem neurobiológica e multidisciplinar. Porto Alegre: Artmed; 2006.

Paín S. Diagnóstico y tratamiento de los problemas de aprendizaje. Buenos Aires: Nueva Visión; 1983. 
Parush S, Yochman A, Cohen D, Gershon E. Relation of visual perception and visual motor integration for clumsy children. Perceptual and Motor Skills; 1998, 86: 291295.

Polat U, Ma-Naim T, Spierer A. Treatment of children with amblyopia by perceptual learning. Vision Research; 2009, 49: 2599-2603.

Rief SF, Heimburge's JA. How to reach and teach all children in the inclusive classroom: practical strategies, lessons, and activities. San Francisco: Jossey-Bass; 2006, 2.

Sattler JM, Weyandt L. Specific learning disabilities: assessment of children, behavioral and clinical applications. San Diego: Jerome M. Sattler Publisher; 2002; 4: 281-335.

Sattler JM. Assessment of visual motor perception and motor proeficiency: assessment of children, behavioral and clinical applications. San Diego: Jerome M. Sattler Publisher; 2002, 4.

Scheiman MM, Gallaway M. Optometric assessment visual information processing: optometric management of learning related vision problems. California: Mosby Elsevier; 2006, 2.

Scheiman MM, Rouse MW. Optometric management of learning related vision problems. St. Louis: Mosby-Elsevier; 2006, 2.

Schneck C. Visual perception. In: Case Smith J. Occupational therapy in children. Missouri: Mosby; 2005, 5: 412-446.

Scott M. School readiness: a head start for preschool years (parent's guide). Pinelands: Pearson Education; 2003.

Sousa DA. How the brain learns to read. California: Corwin Press; 2005.

Tallís J, Soprano AM. Neuropediatría, neuropsicologia y aprendizaje. Buenos Aires: Nueva Visión; 2003.

Vasquez DCO, Cuervo JEO. Concordancia entre dos tests visuo perceptuales MVPT y TVPS en niños clínicamente e metropes entre 5 y 6 años en distintos colegios de Bogotá.Bogotá: Universidad de La Salle; 2009.

Warren M. A hierarchical model for evaluation and treatment of visual perceptual dysfunction in adult acquired brain injury, part. 1. American Journal of Occupational Therapy. The American Occupational Therapy Association; 1993. 47: 42-54.

Williams HG. Perceptual and motor development. New Jersey: Prentice-Hall; 1983.

Wurtz RH, Kandel ER. Vias visuais centrais. In: Kandel ER, Schwartz JH, Jessell TM. Princípios de Neurociência.São Paulo: Manole Saúde; 2003.4(27): 523-547. 
Xiao LQ, Zhang JY, Wang R, Klein SA, Levi DM, Yu C. Complete transfer of perceptual learning across retinal locations enable by double training. Current Biology. California: Elsevier; 2008.18: 1922-1926.

Zhong-Lin L, Yu C, Sagi D, Watanabe T, Levi D. Perceptual learning: functions, mechanisms, and applications. Vision Research. California: Elsevier; 2010. 50: 36536

Artigo recebido em: 08/10/2018

Artigo aprovado em: 16/04/2019

Artigo publicado em: 22/04/2019 